则 $\left(l_{R^{1}},+,-, \cdot, \div\right)$ 与 $\left(F l_{R^{1}}, \oplus, \ominus, \odot, \odot\right)$ 同 构.

我们研究了 Fuzzy 数的分析性质及其应用, 得
到测度、积分和度量空间的若千较为深刻的结果。

赵汝怀

（中国人民武装䇾察部队技术学院光电系, 西安)

\title{
Hayman 定理的简单证明
}

设 $f(z)=z+\sum_{n=z}^{\infty} a_{n} z^{n} \in S$, Hayman 证明了下

面著名的定理：对任何 $f \in S,\left\|a_{n}|-| a_{n+1}\right\| \leqslant K$, 其中 $K$ 为绝对常数。但该证明很长且欠直观. 我们 在这里给出一个简单证明.

设 $f(z) \in S, \log \frac{f(z)}{z}=2 \sum_{n=1}^{\infty} r_{n} z^{n}$, 则对于固定 $r$, 存在实数 $t_{r}$ 使得

$$
\begin{aligned}
& \sum_{n=1}^{\infty} n\left|r_{n}-\frac{e^{i \iota} r^{n}}{2 n}\right|^{2} r^{2 n} \leqslant \frac{1}{4} \\
& \cdot \log \frac{1}{1-r^{2}}+\log (1+r),|z|=r .
\end{aligned}
$$

此式的证明极易从面积原理得出（参见 Sib. Math Zburnal,22, 2: 149-157)

记

$$
\begin{aligned}
& \left(1-e^{i t r z}\right) \frac{f(z)}{z}=1+\sum_{n=1}^{\infty}\left(a_{n+1}-e^{i t_{r} a_{n}}\right) z^{n} . \\
& \log \left(1-e^{i t r} z\right) \frac{f(z)}{z}=\sum_{n=1}^{\infty}\left(2 r_{n}-\frac{e^{i r_{r} n}}{n}\right) z^{n} .
\end{aligned}
$$

由米林-刘别杰夫不等式

$$
\left[\left|a_{n+1}-e^{i t} r a_{n}\right| r^{n}\right]^{2}
$$

$$
\begin{aligned}
& \leqslant \exp \left\{\sum_{k=1}^{n} k\left|2 r_{k}-\frac{e^{i / r^{k}}}{k}\right|^{2} r^{2 k}-\sum_{k=1}^{n} \frac{1}{k}\right\} \\
& \leqslant \exp \left\{\sum_{k=1}^{\infty} k\left|2 r_{k}-\frac{e^{i / r^{k}}}{k}\right|^{2} r^{2 k}-\sum_{k=1}^{n} \frac{1}{k}\right\} \\
& \leqslant \exp \left\{\log \frac{1}{1-r^{2}}+4 \log (1+r)-\sum_{k=1}^{n} \frac{1}{k}\right\} \\
& =(1+r)^{3} \exp \left\{\log \frac{1}{1-r}-\sum_{k=1}^{n} \frac{1}{k}\right\} .
\end{aligned}
$$

因 $\sum_{k=1}^{n} \frac{1}{k}>\log \left(n+\frac{1}{2}\right)+c, c$ 为欧拉常数. 取

$$
r=1-\frac{1}{n}
$$

便得

$$
\begin{aligned}
& \left(\left|a_{n}\right|-\left|a_{n+1}\right|\right)^{2} r^{2 n} \leqslant(1+r)^{3} \frac{n}{n+\frac{1}{2}} e^{-6} \\
& || a_{n}|-| a_{n+1}|| \leqslant 2^{3 / 2}\left(1-\frac{1}{n}\right)^{-n} e^{--c / 2} \leqslant K
\end{aligned}
$$

胡 克 （江西师范大学,南昌）

\section{低温凝聚 $\operatorname{lnSb}$ 膜的相变、亚稳中间相的超导 电性和时间效应}

在亚稳非晶态到稳态结晶态的结构相变过程 中，往往存在着一系列的亚稳中间相. 在这种相变 过程中, 总是伴随着强烈的结晶点阵不稳定性的. 因 此, 研究非晶-结晶相变过程, 及其相变过程中所存 在的亚稳中间相的超导电性，将是高温超导电性研 究中的重要课题, 并且它也应该能为超导电性与结 晶点阵不稳定性之间的关系的研究提供重要信息.

作者及其合作者曾经研究了底板温 度在 57$110 \mathrm{~K}$ 的低温凝聚 $\mathrm{InSb}$ 膜的相变以及新鲜凝聚膜的 超导转变温度与凝聚底板温度之间的关系. 本文主
要研究了低温凝聚 InSb 膜的结晶相变 过程, 并且 获得了以下重要结果:（a）低温凝聚膜从发生第一 电导跃变以后到第二电导跃变发生之前, 样品处于 同一的非晶金属态, 并具有相同的超导 $T_{c}$; (b)发生 第二电导跃变且达到峰值时, 所对应的金属相具有 最高的超导 $T_{c} ;$ （c) 随着第二电导峰值后的电导 下降, 样品的 $T_{c}$ 降低, 且在 $R(T)$ 超导转变中, 样品 由完全的超导电性逐渐过渡到部分的超导电性. 最 后讨论了产生上述结果的物理过程和图象.

本文还研究了低温凝聚膜相变过程中的时间效 